\title{
Apuntes sobre la enseñanza de la historia de la educación física: su articulación con la sociología y la epistemología en la formación de educadores corporales
}

Notes on the teaching of the history of physical education. Its articulation with sociology and epistemology in the training of corporal educators

\author{
León Jaime Urrego*
}

Obrar contra el tiempo y así sobre el tiempo a favor de un tiempo futuro. Nietzsche

\section{Resumen}

Palabras clave: Enseñanza, historia de la educación física, sociología, epistemología y formación de educadores corporales.

\section{Abstract}

El reto puede ser asumido retomando y haciendo actuales las razones que admiten la elección y permanencia de los contenidos que relacionan la educación física con la historia y la epistemología. Digo desafío porque la calidad y presencia de este tipo de contenidos se ven amenazadas como lo denuncian varios autores y como se ve en los impresos publicitarios de planes de formación de licenciados ${ }^{1}$.

El tema que se expondrá trata de la apertura, de lo indispensable, de las posibilidades y de la labor apropiada de la historia de la educación física como conocimiento que aporta comprensión de la experiencia actual de nuestro cuerpo.

El orden de exposición de las ideas será el siguiente: primero se propondrán los contenidos de la historia de la educación física como un saber articulador, deponente y fundador de conocimientos que condicionan y determinan la formación de los educadores corporales; segundo, se dimensionará la epistemología como reflexión que atañe a toda profesión en educación corporal; tercero, se observarán el compromiso y el alcance de contenidos sociológicos en la formación de dichos profesionales. Estos dos últimos apartes podrían leerse como condiciones de posibilidad a ser comprensibles a partir de una historia de la Educación Física.

The challenge can be assumed taking up again and updating the reasons admitting the choice and lasting of the contents that relate Physical Education to history and epistemology. It is a challenge because the quality and presence of this kind of contents are threatened, as some authors remark and it can be infered from the brochures advertising B. A. education programs.

The opening, the need, and the possibilities of the appropriate role of the history of Physical education as a knowledge that helps understanding our present body experience will be exposed.

The topics will be presented as follows: Firstly, the contents of the history of Physical education as an articulating knowledge, foundational to knowledge that determine the training of corporal educators will be discussed. Secondly, there will be a dimensioning of epistemology as a reflection concerning all professions associated to corporal education. Finally, the commitment and scope of sociological contents in the training of physical education teachers will be exposed.

These last two sections might be read as conditions of possibility that can be comprehensible by means of a history of Physical Education.

Key Words: $\quad$ Teaching, history of physical education, sociology, epistemology and corporal educator training.

Fecha de recepción: Septiembre 28 de 2007

Fecha de aceptación: Octubre 26 de 2007

\footnotetext{
Profesor e investigador de la Universidad de Antioquia, licenciado en Educación Física, doctorando en Didáctica de la Educación Física en la Universidad de Valladolid.

1 El presente texto refleja las indagaciones relacionadas con los cursos doctorales Cuerpo y cultura, dictado por el profesor José Ignacio Barbero en 2006; Historia del currículo, orientado por el profesor Agustín Benito Escolano; los trabajos de campo previos a la tesis que desarrollo actualmente, "La cultura profesional que ha educado al cuerpo: discursos y prácticas investigativas en la educación física colombiana”; y predominantemente la influencia de mi trayectoria (2000-2005) en los trabajos de investigación de la línea Historia de la educación corporal, del grupo Estudios de Educación Corporal de la Universidad de Antioquia.
} 


\section{Contenidos de la historia}

\section{de la educación física}

Afirmar que la historia de la educación física puede articular conocimientos y producir comprensión a los límites de la profesión implica dimensionar el conocimiento histórico y su realización de una forma diferente a la imagen tradicional que de este tenemos.

\section{Una nueva historia}

Iniciando el siglo XXI, en algunos círculos académicos parece irrebatible la importancia del conocimiento de los procesos que han posibilitado la constitución de lo actual. Diría Morey (Foucault, 2005) que lo actual no es lo que somos, sino más bien lo que vamos siendo, lo que llegamos a ser. Conocer cómo vamos siendo para comprender y participar del cómo llegamos a ser es una irrenunciable tarea del ser, un compromiso consigo mismo.

La tarea fundamental es la historia, que no solo está implicada con el pasado, sino con la memoria, lo presente y lo futuro. En todo momento la sociedad produce verdades que pone en circulación a través de los discursos. Los discursos no tienen una existencia ideal, sino que se materializan en instituciones, en prácticas, en seres (García Gutiérrez, 2001).

Sobre las prácticas discursivas vale la pena recordar que "no son pura y simplemente modos de producir discursos. Están incorporadas en los procesos técnicos, las instituciones, las pautas de comportamiento general, en formas de transmisión y difusión, y en formas pedagógicas que, al mismo tiempo, las imponen y las mantienen" (Foucault citado por Cherryholmes, 1987: 39).

Esta acepción de historia marca claramente la diferencia con las nociones más tradicionales. Se asocia comúnmente con hombres, monumentos, épocas o fechas grandiosas; pero la nueva historia pretende conocer el presente desde el pasado y proyectarse hacia el futuro. Los hechos requieren ser explicados y sometidos al análisis crítico (Henao y Villegas, 2002). Con esta postura se marca claramente la diferencia entre una "historia del dato" y la de las condiciones de posibilidad de lo actual.

Entendida así, permite conocer las diversas formas de subjetivación. Por ejemplo, en los dominios la educación física no sólo se sabe, sino que se participa en los modos contemporáneos de constitución de identidades corporales, los cuales se han forjado en prácticas sociales históricas y contemporáneas. Estos modos bien merecen la pena conocerse y analizarse.

\section{La educación en la nueva historia}

Siguiendo la línea de la historia de las ideas y de las condiciones de posibilidad, la pedagogía intenta comprenderse a la luz de la memoria. A partir de este conocimiento, la fianza su identidad y conocimiento de lo que le es propio.

Una de las consecuencias surgidas de la relación entre historia y pedagogía es la necesidad de ver la escuela, no sólo como sistema de transmisión de conocimientos, sino también como sistema social (Forquín, 1987). De esta comprensión, de la institución escolar como institución social, se "infiere a su vez, la necesidad de pensar las instituciones y valores, cualesquiera que ellos sean, como resultados de un proceso de construcción, como auténticas invenciones sociales" (Cuesta F., 1997).

Se puede ver que la historia tiene un valor educativo porque permite comprender los procesos sociales que constituyen y constituirán las condiciones que harán posible lo por venir.

\section{La educación física, la escuela y la nueva historia}

La escuela como institución no escapa al influjo de los fenómenos sociales y es allí donde se transmiten modos, usos y prácticas de lo corporal.

La historia de la educación física ha permitido evidenciar: los procesos pedagógicos que regulan las conductas corporales de los seres humanos, las prácticas escolares que pretenden producir y reproducir determinadas visiones de mundo, creencias, ideas, sistemas de racionalidad, modos de pensamiento y de significaciones sobre lo corporal.

Pueden observarse y citarse el caso de Medellín y de los trabajos históricos realizados por el grupo de Estudios de Educación Corporal, en los que se puede evidenciar, conocer y comprender los modos, las instituciones y las prácticas que regulan y configuran históricamente valores culturales que determinaron e inciden en la educación física.

Según lo registrado y analizado en dichas investigaciones (García Gutiérrez, Franco, Vásquez y Urrego Duque, 2002), la educación física del siglo XIX en Medellín perseguía los siguientes intereses: educar a un hombre que relacione su cuerpo con valores morales de conducta; instruirlo a fin de que siga una postura corporal acorde con la disciplina e institución militar; adiestrar a un ser que atienda el cuidado de su cuerpo a partir de prescripciones higiénicas y saludables; y educar a un 
ser para que guarde proporciones entre el trabajo intelectual y el físico, es decir, para que actúe conforme la lógica del ser humano dual (cuerpo-mente).

En suma, acceder a la historia de la educación física es aproximarnos a las prácticas que han constituido la experiencia actual de nuestro cuerpo, y conocer las instituciones y valores que regulan, producen y reproducen determinadas visiones de mundo. Esta aproximación y este conocimiento histórico, asumidos en los términos acá propuestos, permitirían formular la pregunta epistemológica sobre la educación física.

\section{Los contenidos epistemológicos en la Educación Física}

El uso común que podría explicar el significado de la epistemología, la ubicaría en un difuso límite entre un estudio del conocimiento o un estudio de la ciencia; básicamente, sería el estudio crítico de las condiciones que posibilitan el conocimiento y respondería el tipo de interrogantes sobre ¿qué, cómo y cuándo podemos conocer?, ¿cómo sabemos que lo que conocemos es verdadero?

El tema así resumido puede ser caricaturesco, pero no considero que sea oportuno en estas líneas discutir sobre la definición más apropiada de la epistemología, pues amerita preparación y espacio. En el presente texto se aceptará una visión del asunto: la tradición francesa da un tratamiento interesante a la reflexión epistemológica cuando le atribuye la posibilidad de estudiar histórica y críticamente los conocimientos, sus métodos y sus resultados.

Cuando el tratamiento depende de la historia y la revisión crítica de los conocimientos depende fundamentalmente de lo acontecido, de lo que esos conocimientos vienen siendo, y a su vez obedece a las condiciones que los posibilitan. Por tanto, el estudio, y la reflexión o las consideraciones epistemológicas tienen como fuente la historia, entendida en las proporciones acá tratadas.

El lugar que podrían ocupar los contenidos epistemológicos en la formación de los profesionales en educación física no debe referirse solo a "emitir definiciones de lo que es o debe ser entendido por educación física; o de defender teorías para delimitar lo que le compete o no a la profesión"; más bien se puede esperar en el nivel formativo un estudio o reflexión histórica y crítica de las prácticas reales y simbólicas ejercidas, que explique nuestra experiencia corporal a partir de lo que viene siendo (Crisorio, 2003).
Sobre las definiciones, las teorías y los objetos de estudio que se han querido adjuntar a la educación física, Crisorio (2003) señala que diferentes proyectos han pretendido incorporarle la lógica científica para fundar epistemológicamente un cuerpo de conocimientos constituido por un objeto de estudio a priori, y "el resultado ha sido una larga lista de objetos que se suponen objeto de la educación física y de títulos que se pretenden más apropiados para designar sus alcances".

Es frecuente encontrar en la literatura especializada artículos, capítulos o tratados enteros sobre clasificaciones, ordenamientos, enfoques, funciones e incluso definiciones que pretenden atribuir niveles de cientificidad a la educación física. Dicha recurrencia puede obedecer a una búsqueda de fundamentación-justificación que otorgue parcelas definidas, campos firmes o espacios claramente delimitados a los diversos problemas de estudio que se heredan (tradicionales), se presentan (alternativos) y se imponen (por agentes externos, o comunidades académicas específicas).

La preocupación manifiesta en dicha búsqueda sigue una idea de conocimiento y verdad inspirada en la tradicional imagen de la ciencia: clara, evidente, neutral, constatable y"concibe un mundo dado, externo al sujeto cognoscente por entero racional y por entero consciente" (Crisorio, 2003).

Para comprender el panorama y la búsqueda epistemológica en la que se han implicado algunos profesionales de la educación física, es necesario dar cuenta de las prácticas reales y simbólicas de esta, de sus relaciones con los contextos sociales e históricos. Los contenidos epistemológicos dependen fundamentalmente de los históricos y los sociológicos.

La tarea epistemológica dista de nominar objetos, métodos, conceptos, verdades o falacias; en la perspectiva acá aclarada (estudiar histórica y críticamente los conocimientos) se acerca más a la reflexión y al análisis de las condiciones por las cuales la educación física viene siendo lo que es.

Esto implica, ineludiblemente, desplazar su territorio tradicional y sus métodos, analizar sus condiciones de existencia, sus leyes de funcionamiento y sus reglas de transformación; observar la forma en que se utiliza en ella el saber científico ( $y$ las otras formas de conocer en ella), el modo de delimitar los ámbitos de investigación e intervención, el proceso de formación de sus objetos de conocimiento y de creación de sus conceptos fundamentales (Crisorio, 2003: 32). 
De este modo, se trata de dimensionar y concebir un fundamento, alcanzar un suelo, un sentido para la educación física, el cual estaría en la riqueza y diversidad de sus prácticas, conceptos y preguntas. El lugar para buscarlo está ubicado temporalmente en la historia y en las condiciones de posibilidad de la misma, es decir, en su construcción social. Veamos ahora cómo aporta la comprensión de los fenómenos sociales a la epistemología a partir de la ubicación histórica de la educación física.

\section{Contenidos sociológicos en la educación física}

Otros contenidos que afectan positivamente la comprensión y las perspectivas de la profesión son los que implican el estudio de los fenómenos sociológicos. En una relación fundamental con la historia de la educación física, que posibilita articularla con la reflexión epistemológica.

Retomando la idea de la necesaria y permanente mirada a las condiciones de posibilidad que ha constituido la experiencia corporal a partir de la educación física, la atención se dirigirá ahora a resaltar el fenómeno de creación, transmisión o reproducción de modos y formas corporales. Estos fenómenos han sido mediados por procesos y construcciones sociales.

Fernández-Balboa y Muros (2006) sostienen que "la conexión íntima que tiene el deporte y la educación física con otras instituciones los hace un 'campo' ideal, para la reproducción ideológica". Dicha conexión tiene como punto crítico el acto que realiza el profesor o entrenador: seleccionar maneras particulares de pensar, de usar, de consumir y de vivir el cuerpo.

Usualmente, cuando recibimos o participamos de una clase de educación física, percibimos sus efectos inmediatos, pero no observamos la trascendencia y las implicaciones del evento. Por ejemplo, no logramos ver qué orden se instaura con una práctica corporal, quiénes se benefician con dicha práctica, ni qué se prentende inaugurar, mantener o borrar del sujeto con dicha práctica.

Las respuestas a estos interrogantes escapan de los actuales dominios profesionales de la educación física, pero su complejidad no es excusa para no abordarlas; más bien la ausencia de estas es la causa de que muchos profesionales conviertan el acto pedagógico, relacionado con los problemas corporales, en un medio que se limita al cumplimiento de un papel de adaptación, amoldamiento y encuadramiento (Taborda de Oliveira, 2003).
Tradicionalmente, la educación física ha asumido la tarea de formar a los individuos en lo relacionado con lo corporal; pero esta formación ha privilegiado -no siempre ha sido así- contenidos y prácticas educativas instrumentales como los ejercicios, los deportes, los test, las competiciones, las pruebas, asumiendo una noción dualista, un tanto limitada, "que se sustenta en el modelo cuerpo-máquina” (Barbero González, 2001).

$\mathrm{Si}$ aceptamos que la tradición está fuertemente arraigada en esta idea, del "cuerpo-máquina", también es necesario considerar que en estos tiempos, cuando las nociones y las experiencias del cuerpo experimentan profundas transformaciones, mutaciones y articulaciones, surgen otros interrogantes frente a la educación física: ¿Los educadores físicos están preparados para trabajar con esta realidad?, ¿se intenta trascender la acción instrumental, terapéutica, clínica o de intervención por actuaciones en los nuevos y vastos escenarios de la cultura corporal?, ¿̇se intentará ir más allá y reclamar como propósito pedagógico la educación, el tratamiento y el estudio de la cultura corporal?, ¿qué nuevos modos de subjetivación, a partir del cuerpo, está capacitado para ver y estudiar el profesional del área?, ¿qué influencia pueden ejercer estas nuevas subjetividades corporales en la educación física?

Con la pretensión de superar esta situación, si se acepta que la educación física es y ha sido condicionada y constituida socialmente, es decir, delimitada fundamentalmente por unas condiciones de posibilidades históricas y culturales que establecen lo que los seres pueden y deben hacer con sus cuerpos; se demanda en la formación de los profesionales contenidos que asuman la complejidad de sus prácticas a partir de su problematización en los niveles históricos, sociológicos y epistemológicos.

De este modo, la formación debe atender y satisfacer la explicación y la comprensión del cuerpo como una construcción social determinada por saberes, creencias y usos. Un propósito sensato podría ser instaurar en los sujetos que la cursan, la capacidad, el poder y las defensas "(capacidad crítica, si se quiere) ante las demandas culturales en torno al cuerpo que proliferan en la sociedad actual". Según Barbero, es objetivo de la educación física desarrollar la capacidad y poder de decisión, comprensión y resistencia del sujeto frente a los fenómenos de la cultura corporal (Barbero González, 2006).

La capacidad a la que se alude en el anterior párrafo se podría describir con el siguiente término: "criterio". Para alcanzar el "criterio" en los profesionales de la Educación Física es indispensable un conocimiento 
profundo, crítico, consciente y sensible de las condiciones sociales en las cuales se instauran sus saberes, de las diversas opciones, perspectivas y respuestas, de las implicaciones futuras al elegir o tomar partido por una postura, de las experiencias anteriores -que no son otra cosa que la memoria histórica-, de las relaciones posibles entre instituciones, conocimientos, sujetos. En suma, para constituir lo que podría llamarse "criterio" es indispensable el conocimiento sociológico.

\section{Reflexión final}

En los escenarios de formación profesional este tipo de discusiones ha caído en la oscuridad de lo obvio, de lo dado por sentado, de lo que no se discute pues la realidad funciona sin una necesaria explicación. Esto

\section{Referencias}

Barbero González, J. I. (2001). Cultura corporal: ¿Tenemos algo que decir desde la Educación física? Ágora para la Educación Física y el deporte, pp.18-36.

Barbero González, J. I. (2006). Educación física y su didáctica. Proyecto docente para pruebas de habilitación como profesor titular. Valladolid: Universidad de valladolid.

Bourdieu, P. F. (1993). Sociology in question. London: Sage.

Bourdieu, P. F. (1974). The school as a conservative force: Scholastic and cultural inequalities. En J. Eggleston. Contemporary research in the sociology of education (pp. 32-46). London: Methuen.

Cherriholmes, C. (1987). Un proyecto social para el currículo: perspectivas postestructurales. Revista de Educación, pp. 31-60.

Chinchilla, V. J. (2005). Los sentidos de la historia de la educación física. Un balance historiográfico al año 2005. II Congreso Latinoamericano de Historia de la Educación Física. Bogotá: Universidad Pedagógica Nacional.

Crisorio, R. (2003). Educación física e identidad: conocimiento, saber y verdad. En V. Bracht y R. Crisorios, La educación física en Argentina y Brasil (pp. 21-38). La Plata: Al Margen.

Cuesta F., R. (1997). Sociogénesis de una disciplina escolar: la historia. Barcelona: Pomares Corredor.

Fernández-Balboa, J. M. y Muros, B. (2006). The hegemonic triumvirate-ideologies, discourses, and habitus in sport and physical education: implications and suggestions. Quest, pp.197-221.

Forquín, J. C. (1987). La sociología del currículo en Gran Bretaña: un nuevo enfoque en los retos sociales de la escolarización. Revista de Educación, pp. 5-29.

Foucault, M. (2005). Un diálogo sobre poder. Madrid: Alianza. demuestra la necesidad de seguir preguntándonos de dónde venimos, a dónde vamos, qué sentido tiene lo que hacemos, para quiénes tiene sentido, qué se funda con nuestro actuar profesional. Estas preguntas, que no son otras que la misma pregunta por la existencia de la educación física, se presentan como modo fundamental de su realización, es decir, su propio existir de cada momento es en una actitud permanente de compresión de las interacciones del mundo, los seres y los fenómenos que las afectan.

Una responsabilidad fundamental de un profesional de la educación física es estar atento a lo que tiene entre manos, a poner a la vista lo que no está en la superficie de la vivencia corporal cotidiana de los seres, a formular permanentemente la pregunta por su sentido como profesional y también como ser. (ID)

García Gutiérrez, C. E. (2001). Didáctica de la enseñanza de la Educación física del siglo XIX. Revista Educación física y deporte.

García Gutiérrez, C. E., Franco, S., Vásquez, C. y Urrego Duque, L. J. (2002). Discursos de la educación física del siglo XIX en Medellín. Medellín: Universidad de Antioquia.

García Gutiérrez, C. E., Pulido Quintero, S. M. y Urrego Duque, L. J. (2004). Influencia de las ideas modernas en la educación del cuerpo en el ámbito escolar de los discursos de la Educación Física del siglo XIX en Medellín. Revista Iberoamericana de Educación.

García Gutiérrez, C. E., Pulido Quintero, S. M. y Urrego Duque, L. J. (2004). Influencia de las ideas modernas en la educación del cuerpo en el ámbito escolar de los discursos de la Educación Física del siglo XIX en Medellín. Obtenido de http://www.campus-oei.org/ revista/deloslectores/542Garcia.pdf

Goodson F., I. (1991). La construcción social del currículum posibilidades y ámbitos de investigación de la historia del currículum. Revista de Educación, 295, 7-37.

Henao, H., y Villegas, L. (2002). Módulo 5. Estudio de Localidades. En G. Briones, Especialización en Teoría, métodos y técnicas de investigación social. Bogotá: Icfes.

Pascual, C. (1997). Análsis contextual en la formación del profesorado de educación física. Revista de Educación, pp. 161-178.

Taborda de Oliveira, M. A. (2003). Prácticas pedagógicas de la educación física en los tiempos y espacios escolares: ¿La corporalidad como término ausente? En V. Bracht y R. Crisorio. La educación fisica en Argentina y Brasil. Identidad, desarrollos y perspectivas (pp. 151-158). La Plata: Al Margen. 